\title{
Programa de entrenamiento para el incremento de la fuerza muscular
}

\section{Training program for increasing muscle strength}

\author{
Lenin Mendieta ${ }^{1}$; Fernanda Yaguachi ${ }^{2}$; Karla Toledo ${ }^{3}$ \\ http://dx.doi.org/10.21503/CienciayDesarrollo.2013.v16i2.05
}

\section{RESUMEN}

Los trabajos de fuerza muscular datan desde el origen del hombre, cuando este por supervivencia tuvo que adaptarse a las diferentes épocas de la historia. La era primitiva, en donde la caza y pesca eran actividades protagónicas de su vida cotidiana, la Grecia clásica en donde el culto por la belleza del cuerpo les llevo a realizar trabajos de fuerza muscular, la era Romana, en donde se echó al traste la formación física y se vicio de orgías de comidas y bailes, la era Renacentista en donde empieza a despertar el amor por el trabajo físico y la época actual en donde se trabajan los programas de entrenamiento de la fuerza muscular para una mejora de la calidad de vida de las personas, son épocas que sin duda alguna han dejado un legado sobre las diferentes formas de trabajar la fuerza muscular en los seres humanos.

Palabras claves: Programa de Entrenamiento, Fuerza Muscular, calidad de Vida.

\section{ABSTRACT}

The muscle strength works date from the origin of man, when for survival had to adapt to the different periods of history. The primitive era, where hunting and fishing were protagonists activities of daily life, the ancient Greece, where the cult of the body beautiful led them to perform muscular work, the Roman age, where the physical training got lost, and it became addicted to food and dancing orgies, the Renaissance where was beginning to awaken a love for physical work and the current era where work muscle strength training programs for improving the quality of people life; all they certainly have left a legacy on the different ways of working muscle strength in humans.

Keywords: Training program, muscle strength, quality of life.

\footnotetext{
1 Doctor, Mgs Actividad Física y Salud, Mgs Docencia Universitaria e Investigación Educativa, Profesor de Natación de la Real Federación Española de Natación, Profesor Internacional de Fitness Acuático, Investigación en nonagenarios, Madrid, 2010. E-mail: leninmendietatoledo@hotmail.com

2 Doctora en medicina general y pediatría, médico tratante del departamento de pediatría del hospital del seguro social de la ciudad de Loja.

3 Laboratorista del hospital Kockichi Otani de la ciudad de Loja, parroquia Vilcabamba.
} 


\section{INTRODUCCIÓN}

Los programas de entrenamiento de la fuerza muscular están de moda en todos los centros de fitness a nivel mundial como complemento del mejoramiento de la función cardiovascular. Sin duda es importante trabajar la parte aeróbica de los seres humanos para mantener en óptimas condiciones el músculo cardiaco, más es incuestionable la importancia de la fuerza muscular como capacidad principal en el desenvolvimiento de las personas en el transcurso de sus vidas. Axel Gottlob, (2008) indica algunos efectos del entrenamiento de la fuerza que es necesario señalar: Aumento de la fuerza; aumento del perímetro muscular; movilidad funcional; incremento de la velocidad; aumento de la densidad ósea; fortalecimiento de los tendones, ligamentos, fascias y estructuras articulares; aumento de la estabilidad articular; mejora de la postura; mejora de las funciones de protección; mejora del aporte de nutrientes a las estructuras articulares; mejor recuperación después de sufrir lesiones o patologías; mejora de la figura; mejora del aporte metabólico y energético;

Capilarización; mejora de los parámetros cardiovasculares; efectos hormonales beneficiosos

Para poder determinar un programa de fuerza muscular es necesario establecer las definiciones que sobre fuerza existen:

Fuerza general es la fuerza de todos los grupos musculares, con independencia de la modalidad deportiva practicada, y fuerza específica es la forma de manifestación típica de una modalidad determinada, así como su correlato muscular específico. Además existen fuerzas máximas, rápidas y resistencia de la fuerza.

El estudio consistió en diseñar un programa de entrenamiento de la fuerza muscular a trabajar con un grupo etario de 20 a 24 años, estudiantes de la Universidad Nacional de Loja, en la carrera de Cultura Física y siguiendo las normas éticas de Helsinki, modificadas en Seúl 2008 por última ocasión, se hizo énfasis en los beneficios de un programa para el incremento de la fuerza muscular, partiendo desde el campo del diseño curricular y estableciendo una trilogía entre la Educación-Actividad Física-Buen Vivir (calidad de vida).

El Buen Vivir, es más que una buena redacción escrita para la lectura, forma parte de una necesidad buscada en el arjé mismo de nuestra idiosincrasia indo americana, de nuestra identidad pluricultural, un identidad que rompe las barreras de la patria grande y se asienta en la vera de nuestra latino américa, y en los principios fundamentales de la UNESCO, que señala que todos tenemos derecho a una vida digna con calidad de vida.

Según cifras de la Organización Mundial de la Salud, "La inactividad física (sedentarismo) ha sido identificada como el cuarto factor de riesgo para la mortalidad mundial, provoca el $6 \%$ de las muertes en el mundo". La misma fuente señala "En el año 2008, cerca del 31\% de los adultos mayores de 65 años no eran activos y casi 3,2 millones de muertes por año fueron causadas por el sedentarismo".

Los índices de supervivencia son elevados, según cita el informe de la CEPALNACIONES UNIDAD de abril de 2009 (anexo 1 y 2)

En los momentos actuales, se trabaja muy poco los diseños de fuerza muscular, ya que en la mayoría de los centros de cuidado y tratamiento físico, se programa la parte cardiovascular, sin tomar en cuenta que una de las capacidades más 
importante en el desempeño de vida de las personas es la fuerza muscular, las personas tienen un pico máximo de fuerza a los 30 años y de allí en adelante empieza a decrecer de manera lineal descendente, llegando a producirse una pérdida de la fuerza muscular del 1,5\% anual, esta disminución de la fuerza acarrea un desequilibrio de la marcha que se ve acentuado conforme pasan los años, estos cambios dan origen a caídas y, a la muerte de muchos ancianos. Los investigadores de la biomecánica de la marcha en los adultos mayores aciertan al manifestar que una corrección en la marcha disminuiría el número de caídas y que esta se lograría con un trabajo de incremento de la fuerza muscular, lo que se traduciría de igual forma a una mejora de la condición física.

Greenspan SL, Myers ER, Maitland LA, Kido TH, Kresnow MB, Hayes WC, (1989) indican que “...anualmente entre los adultos mayores en los EEUU ocurren sobre 250.000 fracturas de cadera”. Grisso JA, Kelsey JL, Strom Bl, Chin GY, Maislin G, O’Brien LA et al. (1991) indican que “...el 90\% de estas fracturas en los viejos son el resultado de una caída, particularmente entre las mujeres", siguiendo con el estudio de las consecuencias de la debilidad muscular Berg WP, Alessio HM, Mills EM, Tong C, (1997) indican que "...la mayoría de las fracturas de cadera ocurren con relación a la marcha". En un estudio a 407 ancianos realizado por Bennett DA, Beckett LA, Nurrey AM, Shannan KM, Goetz CG, Pilgrim DM et al, (1997), donde se estableció que había una clara relación entre la edad y la posibilidad de caer, encontraron que "...el 13\% de las personas entre 65 y 74 años, tenían algún defecto de la marcha que favorecía el riesgo de caer, así como $28 \%$ de los sujetos entre 75 y 84 años y en casi la mitad de los ancianos mayores de 85 años". Para reforzar lo citado en los estudios clínicos y si se toma en cuenta que los adultos mayores presentan cuatro síndromes que con frecuencia los médicos no dilatan a estudios profundos o no les dan importancia. Estos son según Kane RL,
Ouslander JG, Abass IB, (1994) los 1 amados "gigantes de la geriatría: incontinencia urinaria, demencia, inmovilidad y caídas". La caída definida por la OMS, (1980) como "un evento involuntario que precipita a la persona a un nivel inferior o al suelo" es un problema que se puede evitar y que se presenta de manera frecuente en el adulto mayor. Los porcentajes de caídas anuales en el adulto joven (65-70 años) son según la misma fuente del 25\% incrementándose al 35-45\% en los adultos mayores (80-85 años), disminuyendo a partir de los 85 años el número de caídas, posiblemente por restricción de la actividad física. Se ha comprobado que los adultos mayores frágiles se caen más que los vigorosos (52\% vs $17 \%$ ).

En los estudios realizados en el centro geriátrico Los Nogales de la ciudad de Madrid en el año 2010, por uno de los autores de la presente investigación 11 , se comprobó que existe un incremento de la fuerza del tren inferior del $17 \%$ luego de doce semanas de entrenamiento de la fuerza muscular en ancianos nonagenarios (anexo 3), por tanto se puede inferir que un programa de entrenamiento incrementa la fuerza muscular, a su vez que esta última trae como consecuencia un mejoramiento de la condición física y por supuesto del buen vivir (calidad de vida) en las personas de todas la edades y más aún en los adultos mayores, se considera que este trabajo de investigación es una necesidad social que hay que resolver en la edad joven y continuar longitudinalmente hasta la edad adulta.

\section{Objetivos}

\section{Objetivo general}

- Lograr el mejoramiento del Buen Vivir de las personas a través de un programa de entrenamiento para incrementar la fuerza muscular.

\section{Objetivos específicos}

- Diseñar un programa entrenamiento de la fuerza muscular de las personas. 
- Evaluar el programa de entrenamiento determinada en la fuerza muscular de los estudiantes del cuarto año de la carrera de Cultura Física del Área de la Educación, el Arte y la Comunicación de la Universidad Nacional de Loja.

- Evaluar el programa de entrenamiento determinada en la condición física (calidad de vida)de los estudiantes del cuarto año de la carrera de Cultura Física del Área de la Educación, el Arte y la Comunicación de la Universidad Nacional de Loja.

\section{Hipótesis}

- Con la aplicación de programas de entrenamiento se mejora significativamente la fuerza muscular de las personas.

La variable dependiente es la Fuerza muscular y la Variable independiente es el programa de fuerza muscular.

\section{MÉTODO}

Determinación de la muestra. La muestra fueron los alumnos del cuarto año de la carrera de cultura física del área de la educación, el Arte y la Comunicación, el tamaño de la muestra fue de 21 alumnos, se calculó por medios de procedimientos estadísticos, con ello quedó asegurado un error relativo del $5 \%$ y un nivel de confianza del 95\%.

El programa es diseño de los autores (anexo 4), la intervención se realizó en el gimnasio de la Carrera de Cultura Física de la Universidad Nacional de Loja, utilizando materiales para la aplicación del diseño como son: máquina multifunción de fuerza de resistencia variable de la marca Tecnogym, de fabricación Alemana; prensa de banca para piernas, se resistencia variable, de fabricación artesanal; prensa de banca para brazos, de resistencia variable, de fabricación artesanal; mancuernas de resistencia variable, de fabrica- ción artesanal; escalones de $50 \mathrm{~cm}$ de alto; cajón (step) de $15 \mathrm{~cm}$ de altura por sesenta de ancho y cuarenta de largo, de fabricación artesanal; Hangrip (pinza) de resistencia variable; Barra horizontal; de fabricación artesanal; Ordenador de mesa, marca HP, con programa operativo Windows 7; Sofware para la prescripción médica del ejercicio de diseño del investigador. El clima de la ciudad de Loja fue frío húmedo y lluvioso durante la duración de la investigación y aunque el tratamiento se lo realizo dentro del gimnasio, este no fue climatizado, la temperatura promedio de

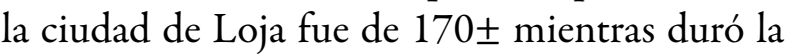
investigación. El diseño del programa de fuerza muscular sigue el esquema del Modelo Piramidal Ascendente, el mismo que está diseñado para el entrenamiento del grupo etario de la presente investigación (20-24 años), con cargas iniciales del $70 \%$ de la fuerza máxima de cada participante y a un 70\% máximo de la Frecuencia cardiaca máxima, que se considera en el rango de moderado según Karvonne, (1963), incrementándose las cargas de acuerdo a la evolución individual del paciente intervenido y reduciendo el número de repeticiones, las repeticiones con las que se empezó el programa fueron en un 1RM calculada con la ecuación de Brzycki, (1993) 1RM= Kg/ $\left(1,02780,0278^{*}\right.$ rep); el programa se diseñó para trabajar los grandes grupos musculares del tren inferior y superior y con el método global de entrenamiento, se trabajó con una frecuencia de tres días a la semana. Alternando lunes, miércoles y viernes, para descansar un día y medio, con un volumen de una hora por sesión y un descanso de 1 a dos minutos entre ejercicio en la primera semana de trabajo, luego de la tercera semana se redujo el descanso a $30^{\prime \prime}$.

Para los criterios de inclusión al estudió se realizaron pruebas clínicas para determinar el colesterol y HDL en el laboratorio 22 del hospital KOKICHI OTANI, las muestras fueron recogidas en el gimnasio de la Universidad a los alumnos; se realizó una evaluación médica, con el fin de determinar el estado de salud física, luego de 
lo cual se introdujeron los datos en un software de prescripción médica del ejercicio (anexo 5) en donde se tomaron datos como peso, talla, sexo, edad, tensión arterial, colesterol, HDL, y se preguntó a los alumnos sobre si ha padecido dolor del pecho, problemas cardiacos, perdida del equilibrio involuntaria, problemas óseos, cirugías recientes, embarazo en las tres primeros meses de gestación, y luego de la revisión médica por parte de una profesional en salud, se pudo constatar que los veintiún alumnos eran aptos para participar del estudio; se extendió un certificado médico individual de cada participante. Para empezar la investigación, se realizó la firma del consentimiento informado por escrito de cada uno de los participantes, en donde dejan señalado que voluntariamente participan en el estudio y que conocen y han sido informados del propósito del estudio.

Se siguió estrictamente las normas éticas de Helsinki, para investigaciones en humanos, modificadas por última vez en Seúl en el 2008.

Se realizó una entrevista con los participantes, los cuales desde el primer momento se encontraron dispuestos a participar en el estudio, se les informó el objetivo de la investigación, de igual manera se realizó una entrevista con el señor Vice Rector de la Universidad, para informarle y solicitarle su aprobación para investigar en el Alma Mater Lojana, el cual estuvo de acuerdo en la realización del estudio.

Se realizó una semana de ambientación al ejercicio lo cual no fue problema ya que al ser alumnos de la carrera de Cultura Física, tenían conocimientos sobre la manera de trabajar, la semana de ambientación se retroalimentaron todos los ejercicios que en el programa se trabajaron, además de establecer un vínculo de confianza con los participantes.

Se realizó un monitoreo de prevención de lesiones y salud en general permanente a los parti- cipantes durante la investigación a cargo de un médico del hospital del Seguro Social de Loja3, la cual extendió un certificado de monitoreo de la investigación y consta en los registros del investigador.

Para la evacuación de datos y su posterior análisis estadísticos, se utilizó el programa SPSS, versión 21, se utilizó un computador HP Compac. Se aplicó una ANOVA de un factor (tiempo) en donde se realizon comparaciones múltiples a posteriori, análisis de varianza de factor de medidas repetidas, en las variables cuantitativas se utilizó las relaciones lineales y el coeficiente de correlación de Pearson. La investigación se trabajó con un nivel de confianza del $95 \%$ y un nivel de significancia del 0,05

\section{RESULTADOS}

Comprobación de las Hipótesis

- Con la aplicación de los programas de entrenamiento se mejora significativamente la fuerza muscular de las personas.

Los resultados de las pruebas de fuerza muscular, evidencian una mejora significativa de la fuerza en las personas que se someten a un programa de entrenamiento, asíse comprueba que la fuerza del tren inferior aumenta significativamente en un $64 \%$.

\section{Cuadros y figuras}

Pruebas tren inferior

Prueba 1. Para fuerza de piernas (Press Banc)

\begin{tabular}{ccc} 
Alternativas & $\begin{array}{c}\text { FUERZA } \\
\text { PIERNAS }\end{array}$ & $\%$ \\
PRE & $63 \mathrm{KG}$ & \\
PROGRAMA & & \\
POST & $103 \mathrm{KG}$ & \\
PROGRAMA & 40 & $64 \%$ \\
\hline DIFERENCIA & 40
\end{tabular}


El cuadro 1. Presentan los resultados relacionados con la fuerza de piernas. Los resultados de esta prueba son altamente significativos llegando a porcentajes de hasta un $64 \%$, en las ganancias de la fuerza luego de la participación en el programa de entrenamiento de la fuerza muscular.

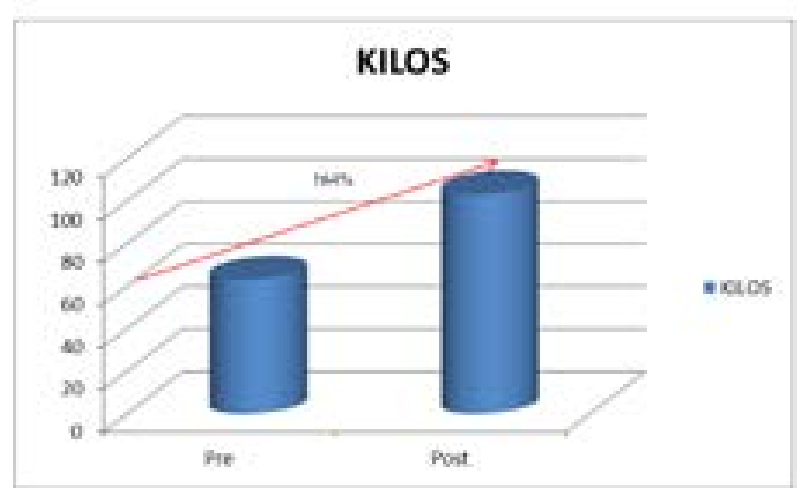

Pruebas tren superior

Prueba 2. Fuerza de brazos (Press Banc)

\begin{tabular}{ccc} 
Alternativas & $\begin{array}{c}\text { FUERZA } \\
\text { BRAZOS }\end{array}$ & $\%$ \\
PRE & 52 & \\
PROGRAMA & \\
POST & 65 & \\
PROGRAMA & & $25 \%$ \\
\hline DIFERENCIA & 13 & \\
\hline
\end{tabular}

El cuadro 2. La prueba de fuerza de brazos, mediante la prensa de banca presenta un incremento del $25 \%$ al finalizar el programa de entrenamiento de la fuerza muscular.

\section{DISCUSIÓN}

Se ha seguido un vector coherente y ordenado en el proceso de construcción del estudio, por tanto es conveniente empezar el recorrido desde la hipótesis planteada "La fuerza muscular se incrementa con un programa de entrenamiento”. A lo cual respondemos que SÍ, que un programa de

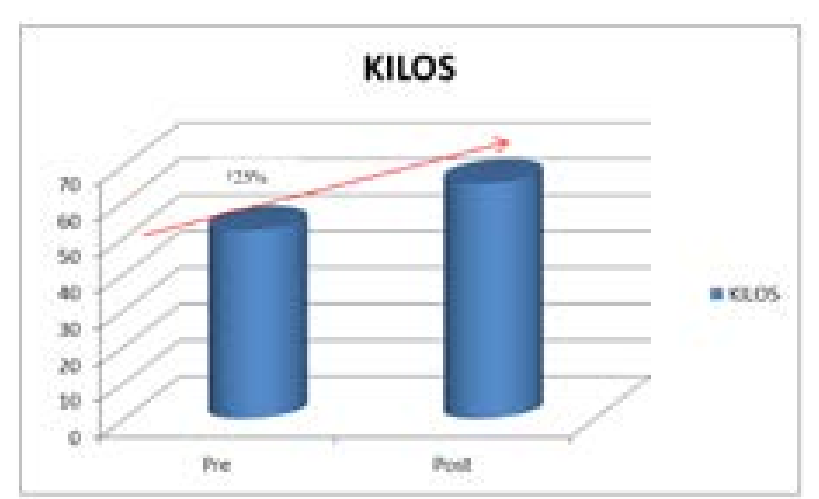

Prueba 3. Fuerza prensión de mano (hangrip)

\begin{tabular}{ccc} 
Alternativas & $\begin{array}{c}\text { FUERZA } \\
\text { BRAZOS }\end{array}$ & $\%$ \\
PRE & 11 & \\
PROGRAMA & & \\
POST & 13 & \\
PROGRAMA & & $18 \%$ \\
\hline INCREMENTO & 2 & \\
\hline
\end{tabular}

El cuadro 3. Los participantes en el estudio de fuerza muscular, tienen ganancias de un $18 \%$ en la fuerza de prensión de manos, mediante la prueba del Hangrip.

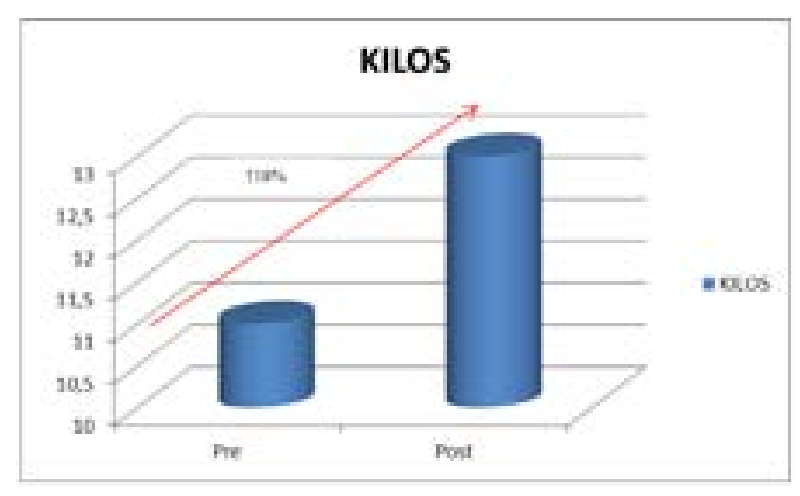

entrenamiento si incrementa la fuerza muscular de las personas. Concordando con los autores, (Cervera Ortiz Vicente. 2006; Corbin. 1987; Fernández Vaquero. 2006; Fiattarone. 2010; Harre, D., M. Hauptman. 1987; Hollmann, W., T. Hettinger. 1980; Kane RL, Ouslander JG, Abass IB. 1994; Kirsch L. 1993; Kuznetsov V. 1989; López Chicharro. 2009; Lucia. 2009; Macchi 
Cuadro 4. Importancia de los programas de entrenamiento de la fuerza muscular.

\begin{tabular}{ll}
1 & Aumento de la fuerza \\
2 & Aumento del perímetro muscular \\
3 & Movilidad funcional \\
4 & Incremento de la velocidad \\
5 & Aumento de la densidad ósea \\
6 & Fortalecimiento de los tendones, ligamentos, fascias y estructuras articulares \\
7 & Aumento de la estabilidad articular \\
8 & Mejora de la postura \\
9 & Mejora de las funciones de protección \\
10 & Mejora del aporte de nutrientes a las estructuras articulares \\
11 & Mejor recuperación después de sufrir lesiones o patologías \\
12 & Mejora de la figura \\
13 & Mejora del aporte metabólico y energético \\
14 & Capilarización \\
15 & Mejora de los parámetros cardiovasculares \\
16 & Efectos hormonales beneficiosos \\
17 & Aumento del rendimiento y de la calidad de vida en personas mayores \\
18 & Mejora del desarrollo y del rendimiento en el niño y en el adolescente \\
19 & Efectos beneficiosos sobre el metabolismo cerebral y sobre la psique \\
\hline
\end{tabular}

G.1993; Menllé. 2010; Nacleiro. 2010; Nati Garcia Villalona, Antoni Martinez y Alfred Tabuenca Monge. 2005; Peréz. 2010) que indican que “...es beneficioso trabajar un programa de mejora de la fuerza muscular, en todas las edades tomando los principios fisiológicos y metabólicos de cada grupo etario".

\section{CONCLUSIONES}

Una investigación de estas características, que ha seguido los parámetros científicos de la investigación en seres humanos y enfocados a la mejora del BUEN VIVIR, acarrea consigo una serie de conclusiones, las cuales se describen a continuación:

Diseñar un programa entrenamiento para el incremento de la fuerza muscular de las personas, es una empresa de muy fácil ejecución si se toman en cuenta parámetros de fisiología del ejercicio, prescripción médica del ejercicio y si se realiza un monitoreo continuo durante el mismo.

En la evaluación del programa de entrenamiento de la fuerza muscular en estudiantes del cuarto año de la carrera de Cultura Física del Área de la Educación, el Arte y la Comunicación de la Universidad Nacional de Loja. Se coincide con la teoría de Jurgen Weineck, (2005) este indica “...la fuerza muscular puede variar entre un veinte por ciento en iniciados y un diez por ciento en entrenados", se refuerza esta teoría con la investigación realizada, en donde se encuentran ganancias de hasta un $25 \%$ en la fuerza de brazos; $18 \%$ en la fuerza de prensión de mano; en la fuerza de piernas de observa ganancias altamente significativas de hasta un $64 \%$ en la prueba del press banc. 


\section{REFERENCIAS BIBLIOGRÁFICAS}

1. Angel. P. Lo sport in Grecia. Roma. Bari, 1988.

2. Astrand y Rodahl. Principales fuentes energéticas del musculo esquelético, Madrid, 2005.

3. Axel Gottlob, Entrenamiento Muscular Diferenciado, Editorial PaidotriboBarcelona, Espaก̃a, 2008

4. Baechle and Earle. Principios del entrenamiento de la fuerza y del ejercicio. National Strength and Conditioning Association, 2007.

5. Bompa, Tudor o, Cornacchia, lorenzo j. Musculación entrenamiento avanzado

6. a los entusiastas de la condicion fisica y a los practicantes de DeportesEditorial hispano europea. 2002

7. Centro Latinoamericano y Caribeño de Demografía (CELADE) - División de Población de la CEPAL, Proyecciones demográficas, 2003.

8. Cervera Ortiz Vicente. Entrenamiento de fuerza y explosividad para la actividad física en el deporte de competición. Tercera Edición, 2006

9. Corbin. Entrenamiento de la fuerza muscular diferenciadad, Stramburgo, 1987. Cornejo. C. Ideario de Rousseau sobre Educación Física. Madrid: Gymnos. 1999. Diem, C. Historia de los Deportes. Barcelona: Luis De Caralt. 1966.

10. Fernández Polcuch E. La medición del impacto social de la ciencia y la tecnología. Argentina. 2000.

11. Fernández Vaquero. Fisiología del ejercicio, Madrid, 2006. Fiattarone. Trabajo de fuerza muscular diferenciada, Australia,

12. Harre, D., M. Hauptmann: "Schnelligkeit und Schnel igkeitstraining”. Theorie und Praxis der Körperkultur, (1987), p198.

13. Hebbling. W. Physical Condition. Trends Trends, 1984

14. Hollmann, W., T. Hettinger: Sportmedizin - Arbeits- und Trainingsgrundlagen. $2^{\mathrm{a}}$ ed., Schattauer, Stuttgart - Nueva York, 1980.

15. Janet Shaw. McGill University B.Ed. (P.E) and the University of Ottawa, Physical and Fitness, 2006
16. Kane RL, Ouslander JG, Abass IB. Essentials of Clinical geriatrics 3rd Ed. New York: Mc GrawHill, 1994.

17. Kirsch L. Entrenamiento isométrico. Ejercicio para desarrollar la fuerza muscular y relajarse. Barcelona: Paidotribo, 1993. pp. 14-15.

18. Kuznetsov V. Metodología del entrenamiento de la fuerza para deportistas de alto nivel. Buenos Aires: Stadium, 1989. pp. 11-13.

19. López Chicharro. Fisiología del ejercicio. $3^{\text {a ed., }}$ Madrid, 2009.

20. Lucía. Fisiología de la fuerza muscular, Universidad Europea de Madrid, 2009.

21. Macchi G. Respuesta cardiovascular a la contracción isométrica. Sport y medicina 1993; 24: 2123.

22. Menllé. Fuerza muscular en pacientes con patologías no adquiridas, Escuela Nacional de Entrenadores de la RFEN, 2010.

23. Nacleiro. Fuerza muscular y entrenamiento deportivo, UEM, 2010.

24. Naglack, Nociones de dinámicas de cargas en el entrenamiento, documentos Biblioteca de UEM 1981

25. Nati Garcia Villalona, Antoni Martinez y Alfred Tabuenca Monge, 2005.

26. Navarro. La Condición Física y los entrenamientos de altura, 1994

27. Ozolin, Sistema contemporáneo de entrenamiento deportivo, 1981. Documentos Biblioteca UEM, 2009.

28. Peréz, M. Fisiología del musculo esquelético, Universidad Complutense de Madrid, 2010 .

29. Peréz, M. Fisiología de los sistemas musculares, Universidad Europea de Madrid, 2010.

30. Pérez Ramírez, Carmen. Evolución histórica de la EF. Revista Apunts, EF, n³3, 24 -38. 1993.

31. Juan Jacobo Rousseau, El Emilio. Traductor, Ricardo Viñas, 2000.

32. Rodríguez, J. J. Historia de la Educación Física, Montevideo, Comisión Nacional de Educación. 1982

33. Zintl, Capacidad de rendimiento, Germany, 1991, traducido UEM, 2007 
34. Greenspan SL, Myers ER, Maitland LA, Kido TH, Kresnow MB, Hayes WC. Trochanteric bone mineral density is associated with type of hip fracture in the elderly. J Bone Miner Res 1994; 9: 1889-94. [Links ]

35. Grisso JA, Kelsey JL, Strom Bl, Chin GY, Maislin G, O'Brien LA et al. Risk factors for falls as a cause of hip fractures in women. $\mathrm{N}$ Engl J Med 1991; 324: 1326-31. [ Links ]

36. Grosser. W.J. Conditión physical and health, Noruega, 1985

37. Berg WP, Alessio HM, Mills EM, Tong C. Circumstances and consequences of falls in independent community-dwelling older adults. Age Aging 1997; 26: 261-8. (Links)

38. Bennett DA, Beckett LA, Nurrey AM, Shannan KM, Goetz CG, Pilgrim DM et al. Prevalence of parkinsonian signs and associated mortality in a community population of older people. N Engl J Med 1996; 334:71-6. [ Links ]

39. Fuente. Organización Mundial de la Salud. Sedentarismo en el mundo, 2012.

40. E. Segura, V. Rodilla, J. F. Listón. Fisioterapia durante la hemodiálisis, resultados de un programa de fuerza resistencia. Universidad de Valencia, 2008

41. Rene González Botto, Las escuelas gimnásticas y su relación con la actividad física y educación física actuales, León, España, 2004

42. Clark, J. La evolución de las Tendencias, , 1967. Documentos UEM, 2009 